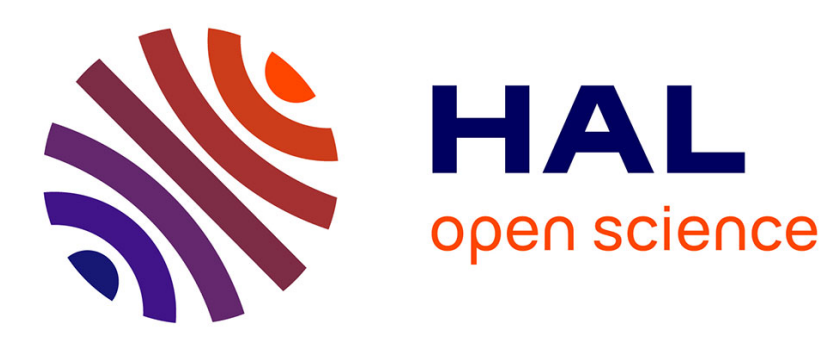

\title{
Synergistic control of a multi-segments vertebral column robot based on tensegrity for postural balance
}

\author{
Artem Melnyk, Alexandre Pitti
}

\section{To cite this version:}

Artem Melnyk, Alexandre Pitti. Synergistic control of a multi-segments vertebral column robot based on tensegrity for postural balance. Advanced Robotics, 2018, pp.1 - 15. 10.1080/01691864.2018.1483209 . hal-01822537

\section{HAL Id: hal-01822537 \\ https://hal.science/hal-01822537}

Submitted on 26 Jun 2018

HAL is a multi-disciplinary open access archive for the deposit and dissemination of scientific research documents, whether they are published or not. The documents may come from teaching and research institutions in France or abroad, or from public or private research centers.
L'archive ouverte pluridisciplinaire HAL, est destinée au dépôt et à la diffusion de documents scientifiques de niveau recherche, publiés ou non, émanant des établissements d'enseignement et de recherche français ou étrangers, des laboratoires publics ou privés. 


\section{Synergistic control of a multi-segments vertebral column robot based on tensegrity for postural balance}

\section{Q1 Artem Melnyk ${ }^{\mathrm{a}}$ and Alexandre Pitti ${ }^{\mathrm{b}}$ \\ a Héphaïstos Project, Université Côte d'Azur, INRIA, France; 'baboratoire ETIS, Université Paris Seine, Université de Cergy-Pontoise, CNRS UMR,} ENSEA, Cergy-Pontoise, France

\section{ABSTRACT}

We present a neuronal architecture to control a compliant robotic model of the human vertebral column for postural balance. The robotic structure is designed using the principle of tensegrity that ensures to be lightweight, auto-replicative with multi-degrees of freedom, flexible and also robust to perturbations. We model the central pattern generators of the spinal cords with a network of nonlinear Kuramoto oscillators coupled internally and externally to the structure and error-driven by a proportional derivative (PD) controller using an accelerometer for feedback. This coupling between the two controllers is original and we show it serves to generate controlled rhythmical patterns. We observe for certain coupling parameters some intervals of synchronization and of resonance of the neural units to the tensile structure to permit smooth control and balance. We show that the top-down PD control of the oscillators flexibly absorbs external shocks proportionally to the perturbation and converges to steady state behaviors. We discuss then about our neural architecture to model motor synergies for compliance control and also about tensegrity structures for soft robotics. The 3D printed model is provided as well as a movie at the address https://sites.google.com/site/embodiedai/current-research/tensegrityrobots.

\section{ARTICLE HISTORY}

Received 11 December 2017 Accepted 17 May 2018

\section{KEYWORDS}

Motor synergies; central pattern generators; tensegrity; vertebral column; postural balance; phase synchronization; soft robotics; feedback resonance; biological robotics

\section{Introduction}

Animal's musculoskeletal system is based on a complex network of muscles, bones, nerves, tissues, and soft bodies which are hard to replicate accurately in robots $[1,2]$. This dense architecture is however well ordered so that the control done in the nervous system can realize easily flexible sensorimotor coordination at a very low energy cost with the dynamical grouping of the muscles known as motor synergies [3-5]. Nonetheless, in order to exploit fully the body structure, the nervous system has to organize itself flexibly and complementarily [6-8]. For instance, the well distribution of stress and strain throughout the body warranties its ecological control so that when we are exposed to a violent shock, we can still stand or bend our knees or stiffen (or soften) our body and joints with a small amount of control, just as a building would absorb an earthquake wave and balance itself, or as a bridge would lean into the wind. In comparison, current robots are still difficult to design and to control in order to achieve robust postural balance under external perturbations and dynamic motion.

In this paper, we propose to take inspiration of both (1) the human musculoskeletal system of the dorsal spine and (2) the neural architecture at the spinal cords level to realize a multi-degrees of freedom vertebral column robot [9-12] and its neural controller in order to cope with external perturbations. Our first contribution is on the one hand on the design of an original neural controller composed of a proportional derivative (PD) controller and nonlinear oscillators in order to generate controlled rhythmical patterns and convergence to steady state behaviors. Our second contribution is on the other hand on the design of a novel vertebral column robot constituted of connected auto-replicative tensegrity elements [13] mounted vertically as a multi-segment inverted pendulum with soft links. To our knowledge, this coupling between a PD controller and nonlinear oscillators to control synchronization as a minimization process was never presented before.

We model the central pattern generators (CPGs) of the spinal cords with a network of nonlinear Kuramoto oscillators coupled internally and externally to the structure and error-driven by a PD controller using an accelerometer for feedback. We suggest that our proposed neural controller, although simple, is similar to the role played by the neuromodulators in the spinal cord that modulate the

CONTACT Alexandre Pitti alexandre.pitti@u-cergy.fr E Laboratoire ETIS, Université Paris Seine, Université de Cergy-Pontoise, CNRS UMR 8051, ENSEA, Cergy-Pontoise, France 
gain of the sensory feedback on the alpha-motor neurons activity to generate the desired synergy [14-16]; which means selecting the most expected resonant modes relative to the perturbation. In line with $[8,17]$, we consider postural coordination modes as emergent phenomena giving rise to non-linearity properties such as phase transition, multistability and hysteresis [18].

The paper is organized as follows. In Section 2, we present first our motivation for the design of a tensile structure. In Section 3, we describe then its assembling with replicated $3 \mathrm{D}$ printed elements, its motors and sensors used in order to replicate the tendon-driven mechanism and control of the human upper-body. We present the neural oscillators used to model the so-called ${ }_{4} \mathrm{CPG}$ and the feedback-driven PD control used to model the neuromodulators. Tested in passive and active conditions, the multi-DOF tensile structure shows a large spectrum of behaviors from very soft dynamics capable to generate rhythmical oscillations to very rigid static postures capable to handle its own weight in every posture.

In Section 4, we propose four experiments to display the capabilities of our framework. In the first setup, we define the dynamics in open-loop control for various modes of coordination by varying the phase and the duration of a pulse-width modulation (PWM) control and by analyzing the resonant frequencies of the system and its rhythmical patterns. In the second experiment, we analyze the system statically from a postural viewpoint and study its robustness in the upward position. In the third experiment, we propose to exploit error feedback for closed-loop control of the structure using CPGs [19]. Depending on the values of the internal and external coupling parameters, we can synchronize nonlinear oscillators modeled with Kuramoto units to the resonant modes of the structure and entrain it freely to specific directions. These privileged modes of synchrony represent the natural motor synergies that are possible to generate and control in the multi-segmented structure [20].

In the fourth experiment, we employ a top-down mechanism, a PD controller, that controls the amplitude level of the oscillators in order to absorb the external perturbations gradually on the vertebral structure so that it can return back to its resting upward posture. Depending on the strength of external perturbation, the controller will set the oscillators to a certain regime producing big oscillations till recovery in order to absorb the shock. In reverse, for tiny perturbations, the controller will set the oscillators to a different regime that can dampen the perturbations.

We discuss then the interest of our mechanical design and of our neural network for controlling soft robots as well as the links to human motor synergies.

\section{State of art and motivation}

One architectural design that explains well biomechanical compliance is tensegrity structures $[21,22]$. Tensegrity structures can be seen as physical networks of stress and loads so that they have an inner stress and plasticity in their structure that make them resilient, adaptive and robust to some external loads. In comparison to most robotic designs, they do not follow Newton's law for rigid bodies as they have no joints and no momentum or torque since the motors are not on the axis of articulation. Instead, they follow Hooke's law for elastic bodies. These features make them a promising paradigm for integrating structure and control design [23-25]. For instance, we can easily formalize a tensegrity system as a network of tension (muscles and soft tissues) and compression (bones), or as a network of springs and masses [26,27]. Therefore, they can be viewed as complex dynamical systems with many degrees of freedom [10,28,29], which is a property often seen in biological systems [30-32].

The redundancy and nonlinearity within such dynamical system might be considered at first as an obstacle to control, however, the symmetries of the overall structure and the many resonant modes generated can serve to decrease the dimensionality of the control problem. For instance, one way to have an adaptive control is to exploit phase synchronization of these modes similar to coupled chaotic maps [7,33].

In human control, this work is achieved by the ${ }_{2} \mathrm{CPG}$ at the spinal cord level, which are primitives that control the muscle grouping for general motion behaviors $[14,34]$. When we are standing upward, for instance, groups of muscles are dynamically selected to contribute to our stability depending on the error perturbation level and the force direction $[35,36]$. In robotics, such bioinspired control is still difficult to model when we design a compliant body with many degrees of freedom and nonlinearities. In previous works, we have shown how we can control such high degrees-of-freedom system with chaotic controllers that sync dynamically to the resonant frequencies of several robotic devices [29,37-39] and to human partners [40]. We believe that this type of control conveys some important features of the human musculoskeletal system control as done in the spinal cord by the CPGs $[34,41,42]$.

\section{Material and methods}

We present a tensegrity structure based on autoreplicative elements, $3 \mathrm{D}$ printed and similar to the ones proposed in $[13,43]$, see Figure $1(a, b)$. This particular 
221

a)

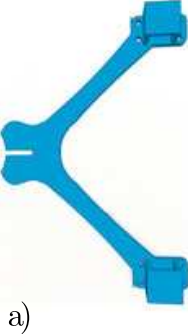

b)

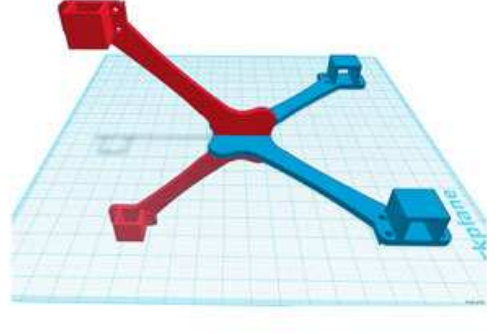

d)

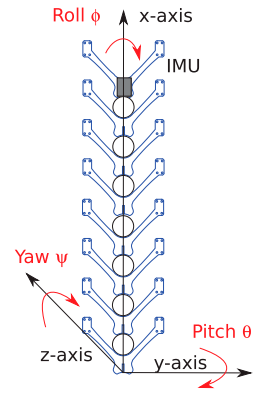

Conception. We display in Figure 2 the prototyping of Figure 1. Multi-joint vertebral column model based on autoreplicative tensile elements for soft robotics. (a-c) Each element forms a tetrahedron by its four edges, which ensures the whole compliance and tension distribution in the three directions when connected to other elements in line. (d) An Inertial Measurement Unit (IMU) is placed at the end of the structure for position control and acceleration feedback. We provide the design of the replicative element freely at the address [44].

motif reproduces the very stable structure of the tetrahedron (i.e. the pyramids) which can stand easily upward, see Figure 1(c,d). When two structures are assembled as two inverted pyramids, the coupled unit structure can move in two directions and can support small sheer torsions as well in the third direction, which is ideal for modeling the human's rotational joints.

In comparison to other types of tensegrity motives, this one has the advantage to require fewer structures and few junctions part. Each element is connected to others with springs, which confers to the design some viscoelastic properties comparable to pre-stressed structures. The tensile elements possess a stable configuration that returns even after applying some external force pressure (self-stabilisation). The whole structure has 10 elements connected with spherical joints (ping-pong balls), which models well the excentric rotations of bones articulation. The vertebral column measures around 1-meter height; and each element is occupying a volume of 11 centimeters cube. The system can be viewed as a threedimensional version of mass-spring dampers mounted in series. The total weight of the robot vertebrae is under $800 \mathrm{~g}$, counting the weight of the motors ( $25 \mathrm{~g}$ each) and of each element $(30 \mathrm{~g})$, which is very light concerning its size. the tensegrity model with motors inserted and springs attached. A better understanding of which part of the robot each motor actuates is provided further in the Muscle-Tendon section Figure 3. To show the properties of compliance and postural stability of the structure, we co-contract the motors and set its neutral postural configuration respectively in the horizontal plane and in upward tension, so that the structure has a maximum momentum and tension on its morphology horizontally and has to exploit its physics fully to stand vertically; see resp. Figure $2(\mathrm{a}-\mathrm{c})$. The balanced forces distribution of each motor-driven cables on the whole structure makes it stabilized in every position, even for the less energetical ones as the horizontal plane or the vertical plane, which are also difficult for humans who develop different strategies to support their own postural balance $[18$, $31,36,45]$.

Sensory acquisition. To perceive the motion, an inertial measurement unit (IMU) is placed at the top of the vertebral column as shown in Figure 1(d). This module possesses one accelerometer and a gyroscope, which permits to measure an angular velocity in $\mathrm{rad} / \mathrm{s}$ and linear acceleration in $\mathrm{rad} / \mathrm{s}^{2}$. Since the Gyro drifts slowly from its position and the accelerometer has high-frequency parasites, we can combine the two information to get rid of the slow variations of the gyroscope (high-pass filter) and the fast variations of the accelerometer (lowpass filter). The equation of the complementary filter for the ${ }_{\perp} Y$ angle is: $\theta_{I M U}=0.98 x(\theta+G y / d t)+0.02 x Y_{a} c c$. Where $Y_{a} c c$ is the angle of the accelerometer in degree and $G y$ the variation of the gyroscope. We mount the IMU at the extremity of the structure to have the maximum amplitude variation as the feedback signal. We plot in Figure 1(d) the $X Y Z$ coordinate system in which the $\mathbf{x}$-axis is opposite to the gravitational field and points upward so that the IMU is aligned along the longitudinal axis of the robot, the $\mathbf{z}$-axis is perpendicular to gravity and lies in the horizontal plane of the robot body and the $\mathbf{y}$-axis is aligned in accordance to both the $x$ - and $z$ axis in order to form a right-hand three-axis coordinate system. The rotations in roll $\phi$, yaw $\psi$, and pitch $\theta$ represent changes in orientation about the $x, z$ and $y$-axes respectively, see Figure 1(d).

Muscle-tendon model. The structure is actuated by six electrical micro-motors with a gear head and a shaft that reel up a $10 \mathrm{~cm}$ tendon-like wire and assembled in opposite sides, two by two, all over the structure and every two segments to have some flexibility (under-actuation).

Using the same the nomenclature proposed by Geyer and Herr [46], we propose to model the system dynamics as actuated mass-spring-dampers connected in parallel and in series. For instance, each tensile element is 
331

d)

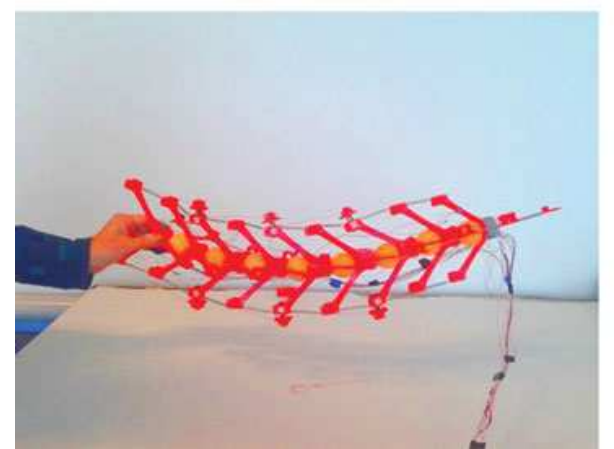

e)

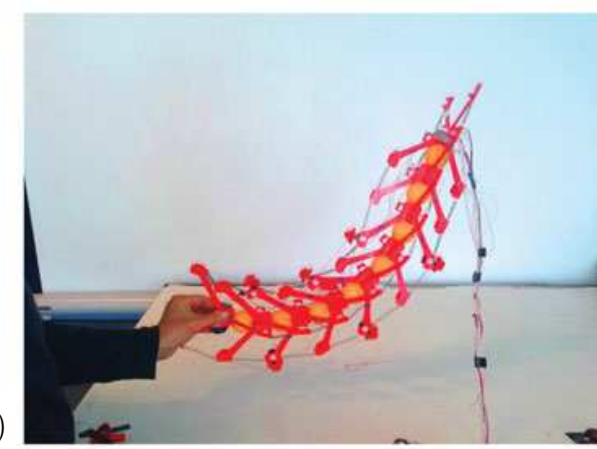

a)

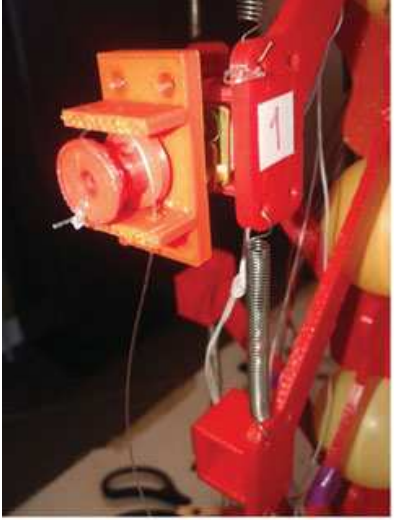

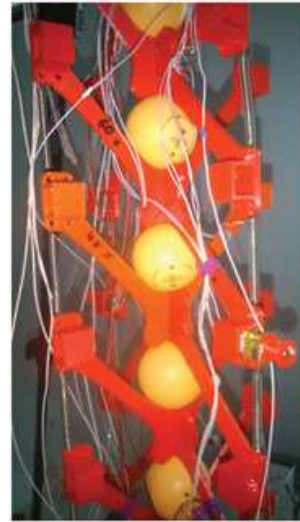

c)

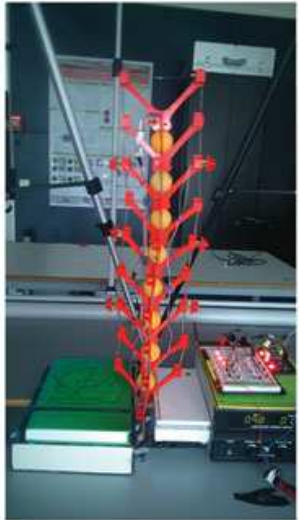

Figure 2. Robustness in co-contraction in horizontal and upward postural configuration. Static postures demand to set the contraction of all the motors to specific lengths. In these situations, the motors act as rigid tendons and loads are distributed overall the structure. Maximal efforts are delivered on the structure when set at the horizontal in (a), in the vertical plane with different directions in (b), and upward at the vertical in (c).

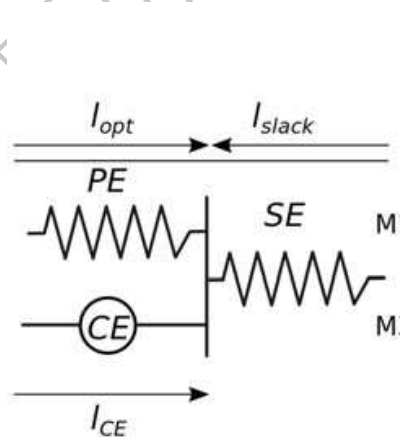

a)

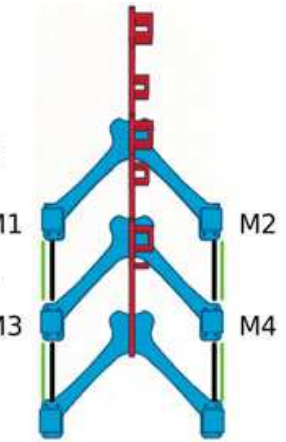

b)

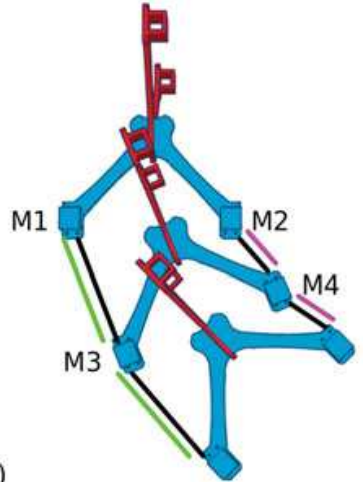

c)

Spring

Wire released

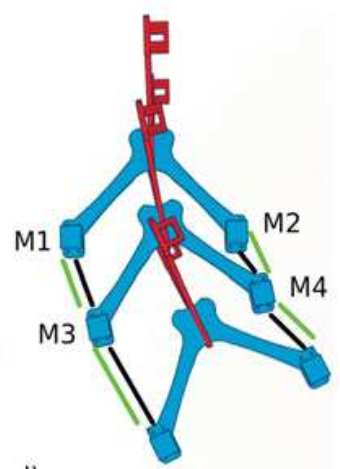

d)

Wire contracted

Q22 Figure 3. Muscle model in co-contraction within the structure. Motors are mounted with springs in parallel and in series on the structure, which ensures the tensegrity system to be always pre-tensed. The motors pairs M1/M2 and M3/M4 are mounted in the opposite direction for co-contraction.

formed with an active contractive element (CE) together with elastic series (ES) springs constituting one muscletendon unit (MTU), see Figure 3(a). Each spring has an optimum length $l_{\text {opt }}$ in which the global system is in a static configuration. if the CE stretches an ES spring beyond its optimum length $\left(l_{\mathrm{ES}}>l_{\mathrm{opt}}\right)$, a parallel elasticity (PE) spring engages in the opposite direction so that its length becomes $l_{\mathrm{PE}}<l_{\mathrm{opt}}$. Conversely, as each tensile unit possesses two actuators mounted in opposition, the opposite PE prevents the ES of abrupt slack. We can now put in equation the system's behavior using these elements depending on the action of CE. Knowing 
the stimulation $S(t)$ of the muscle $m$ and the muscle time delay $(\Delta t)$ and gain $G$, we have $S(t)=S_{0}+G\left(l_{\mathrm{CE}}-\right.$ $\left.l_{\text {off }}\right)(t-\Delta t)$ where $l_{\text {off }}$ is a length offset and $S_{0}$ a stimulation offset.

\subsection{Controllers}

We describe in this section the three different controllers used to explain our original model to control the soft vertebral robot. In the first section, we present the error-driven PD controller and how it is implemented to control the structure as a classical invert-pendulum. In the second section, we present the oscillatory-based Kuramoto network that creates the rhythmical synchronization pattern in open-loop or in closed loop. And in the third section, we present our original approach that combines both approaches, PD+Kuramoto, for rhythmical synchronization and error-based attenuation of oscillations during external perturbation.

$P D$ control. One common way to control the structure in its upward position is to use PD controllers to minimize the proportional (P) and derivative errors (D) based on sensory feedback [46]. The variable (e) represents the tracking error, the difference between the desired input value $(\mathrm{R})$ and the actual output (Y), the angular position computed from the IMU from the vertical axis.

This error signal (e) will be sent to the PD controller, and the controller computes both the derivative of this error signal. The signal (u) just past the controller is now equal to the proportional gain $\left(K_{P}\right)$ times the magnitude of the error plus the derivative gain $\left(K_{D}\right)$ times the derivative of the error: $u=K_{P} e+K_{D} \dot{e}$.

This signal (u) will be sent to the motors fed as a PWM controller, and the new output $(Y)$ will be obtained. This new output $(Y)$ will be sent back to the sensor again to find the new error signal (e). By convention, the motor units on the left side will receive the command (u) whereas the motors on the right side will receive the command (-u). In the following experiments in Section 4, we will control the tensegrity column in one plane only in the $Y$-axis using a PD controller on the motor units or the oscillators respectively in Sections 4.3 and 4.5.

Kuramoto oscillators. The PD control presented in the previous section is driven by error feedback and can serve as an adaptive strategy for stabilization if the proper coefficients are found. In comparison, oscillator-based controllers can model rhythmical patterns that can improve the control of speed as well as robustness against noise when the proper feedback-driven coupling is found. We propose to model also this second type of controllers for the balance and postural control of the tensegrity column.
The archetypal limit-cycle oscillators are the van der Pol or Fitzhugh-Nagumo ; however, we adopt in our experiments the adaptive Kuramoto oscillator for its simplicity to control the motor units. Each oscillator has its intrinsic frequency and can receive interoceptive signals either from other oscillators or descending controllers and exteroceptive signals (in our case from the IMU sensor), see Figure 4 the blue lines. Depending on the Q2. coupling parameters for internal and external feedback, the oscillators can have a phasic adaptation to the robot motion -, which can serve to accelerate it or to damper it concerning the oscillator's own pace,- or an abrupt change to a non-rhythmic behavior.

Each oscillator $\vartheta$ has its own intrinsic frequency $\omega$, and each one receives a linear combination from internal and external dynamics. The internal coupling is done by computing the phase difference between the oscillators. The external coupling is done similarly by computing the phase difference between the sensory feedback from the IMU unit, $\theta_{\text {IMU }}$ and the current phase of the oscillator $\vartheta_{i}$. We define the three coupling coefficients, KI, KE and JE corresponding, respectively, to the internal coupling among the oscillators, the external coupling of the external signal to the units and the amplitude level of the output signal to the motors. The internal coupling KI synchronizes the oscillators from each other and reduces their variability. The external coupling KE influences the coupling to external perturbations. Besides, the control of the amplitude signal to the motors JE is used to perturb

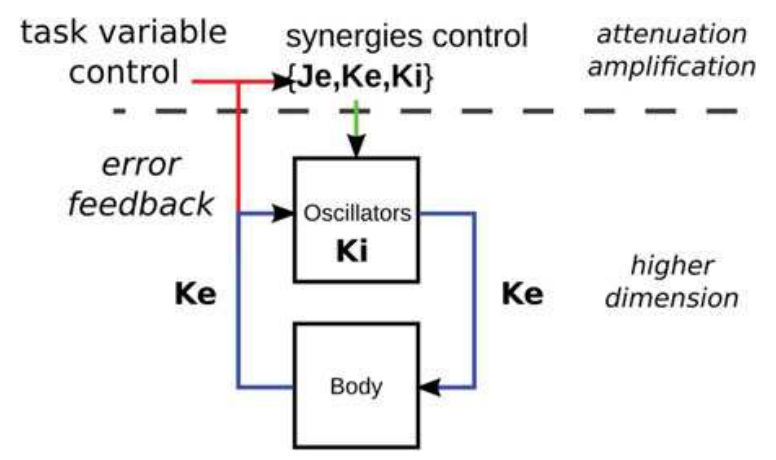

Figure 4. Hierarchical control based on top-down PD controllers and bottom-up oscillators. The whole control of the motor synergies is based on the coupling parameters set $\{\mathbf{K E}, \mathbf{K I}, \mathbf{J E}\}$. The Kuramoto oscillators are coupled internally to each other based on the parameter KI. The external coupling is done by the parameter $\mathbf{K E}$, which sets the influence of the IMU sensor on the internal dynamics. The parameter JE acts on the amplitude of the Kuramoto units. The rhythmical patterns produced by the oscillators can be controlled by a higher center of decision that selects the global parameters for a desired postural control. This hierarchical control corresponds to a dimensionality reduction of the controller on the robot dynamics to generate a feedback-based closed loop control on oscillatory movements. which are employed in many robotic experiments to model the spinal cord's ${ }_{4}$ CPGs $[42,47,48]$. 


\section{a)}

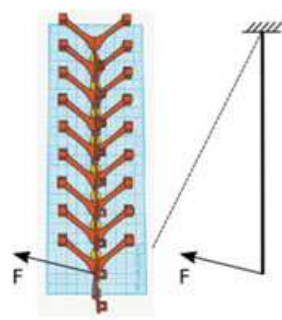

$P D$ control on the Kuramoto oscillators. The parameters $\{\mathbf{K I}, \mathbf{K E}, \mathbf{J E}\}$ set the oscillators to certain rhythmical regimes, which can be viewed as imposed motor synergies [5]. Therefore, one way to combine the rhythmically based control with the reflexive-based control presented before is to put atop of the oscillators a PD controller to modulate the values of the parameters set $\{\mathbf{K I}, \mathbf{K E}, \mathbf{J E}\}$. In comparison, the PD controllers atop of the Kuramoto network can modulate its parameters to tune the motor synergies or to repel them from discrete patterns. Therefore, the control of this parameter set $\{\mathbf{K I}, \mathbf{K E}, \mathbf{J E}\}$ may be seen similar to the descending neuromodulators in the spinal cord that control the activity level of the alphamotor neurons.
This compound controller will drive directly the oscillators toward desired regimes by changing the coupling parameters based on error feedback; the new circuit is plotted in red links in Figure 4. We use for that the dis- Q3. tance to the vertical $Y_{\text {err }}$ as a measure of the error $e$ to synchronize the oscillators to the amplitude level of the perturbing force by updating the parameter JE with the PD equations such that $\mathrm{JE}=K_{P} Y_{\text {err }}+K_{D} \dot{Y}_{\text {err }}$. Such a strategy may be similar to the task-level commands performed by the neuromodulators in the higher centers of decisions to bypass or to activate the synergistic control at the spinal cord leyel $[4,5,15,35]$.

\section{Results}

We present in this section the results that characterize our soft robot with perturbations in passive mode in order to retrieve ack its fundamental resonant fre- Q4 quencies (Sections 4.1 and 4.2) and in active mode to control the multi-segments structure for balance and standing upward based on error feedback and oscillations nearby its intrinsic frequencies (Sections 4.3, 4.4 and 4.5).

\subsection{Dynamic behavior analysis in passive conditions}

We make first a perturbation analysis of the whole structure and present its dynamic behavior when pushed in the frontal plane and when pushed on the transverse plane, respectively (Figure 5(a,b)). Specifically, we are interested in the passive response under external stresses and the response of the controllers.

C)

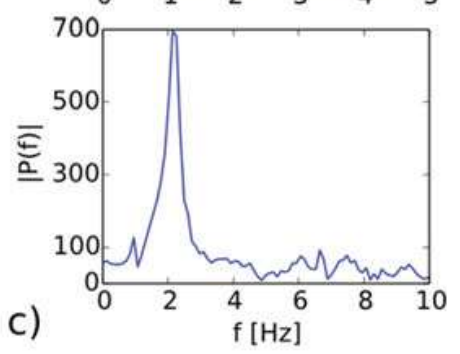

Figure 5. Perturbation analysis on the vertical and axial plans of rotation, resp. top chart and bottom chart in (a) and (b). The Fourier transforms in (c) show the resonant frequencies of the structure. Two different fundamental resonant frequencies are found for the two axis, respectively $0.5 \mathrm{~Hz}$ and $2 \mathrm{~Hz}$. 
a)
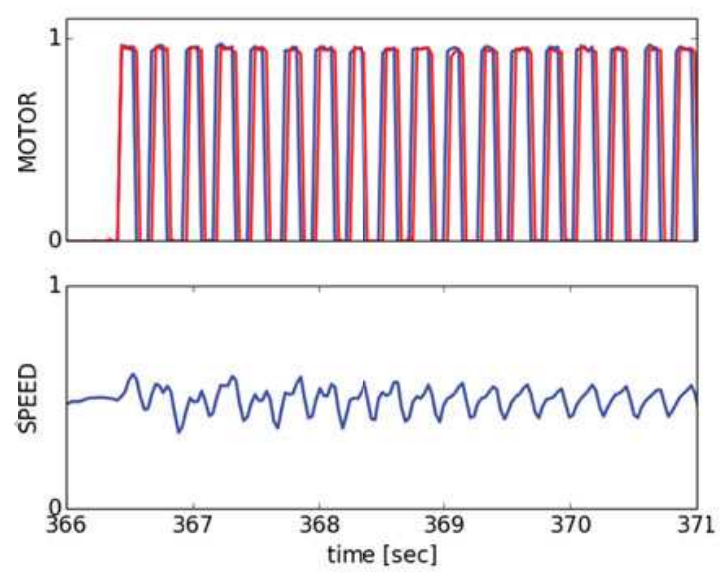

duration

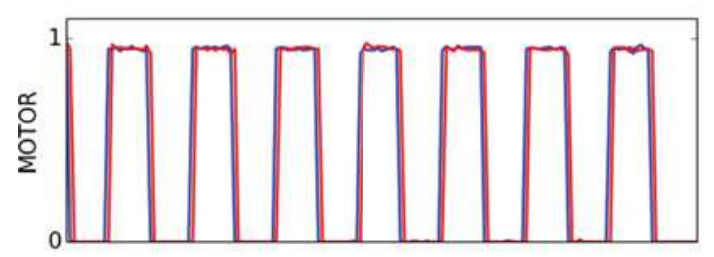

c)

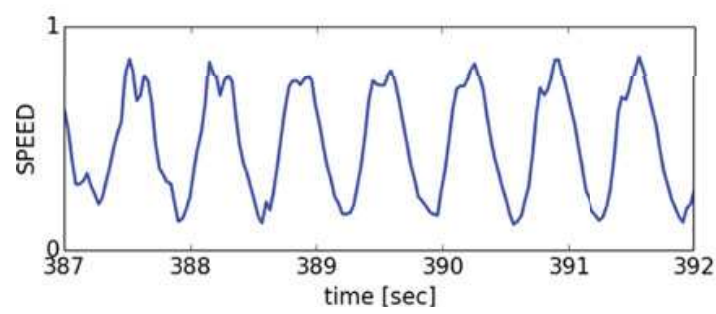

\subsection{Resonant mode analysis in controlled} conditions

We propose to study in this section the control of the different resonant modes and phase delays of the structure with open-loop controllers using PWM controllers. To facilitate the analysis, we group the motors aligned symmetrically in the longitudinal plane forming two clusters of three motors each. We control the phase delay and duration of the PWM between the two motor groups around the fundamental frequency.

If we modulate the duration of the PWM controllers for all the motors as in Figure 6(a-d), respectively, from 50. 100, 25 and 500, ms, we can observe sensitivity on the oscillatory patterns of the tensegrity structure. As we might expect, large PWM produces large amplitude oscillations whereas small periods of the PWM generate weak perturbations. The resonant mode occurs for 250 $\mathrm{ms}$ period square signal with amplitude variation 3 times larger than for PWM of 50. ms. Above this value,
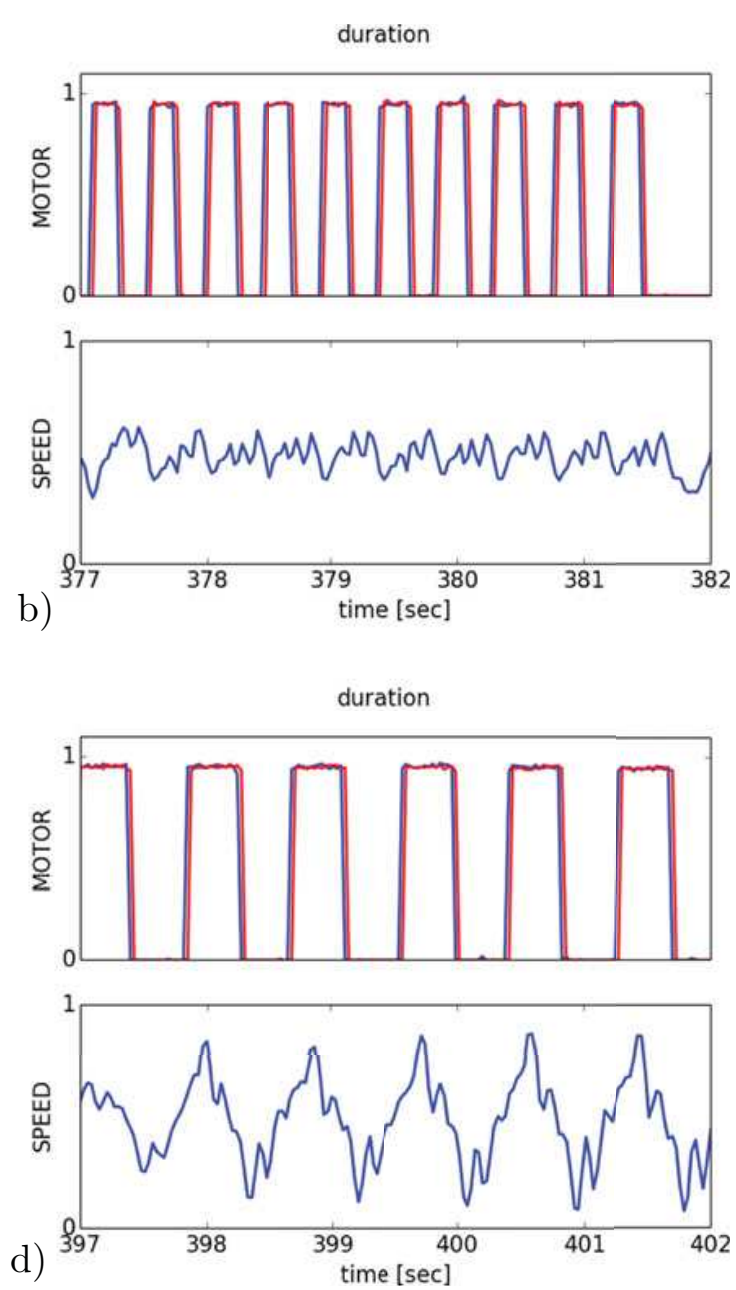

Figure 6. Phase duration characterization in controlled condition with PWM. A PWM signal controls the tensegrity structure in the vertical axis with motors aligned along the vertical axis. Modulating the phase duration of the motors, from small durations $50 \mathrm{~ms}$ (resp (a), $100 \mathrm{~ms}$ (resp (b), $250 \mathrm{~ms}$ (resp (c) up to $500 \mathrm{~ms}$ (resp. (d), affects the level of global synchrony and the apparition of complex modes of resonance. 
in the case of Figure 6(d), new harmonic modes are super-imposed on the speed signal, which corresponds to complex modes of coordination with the apparition also of harmonic waves.

\subsection{PD control}

We perform first the error-based PD control of the upward structure relative to the $Y$ axis with directly $e=Y_{\text {err }}$, the relative displacement. The PD controller can be written as $u=K_{P} Y_{\text {err }}+K_{D} \dot{Y}_{\text {err. }}$. We present a

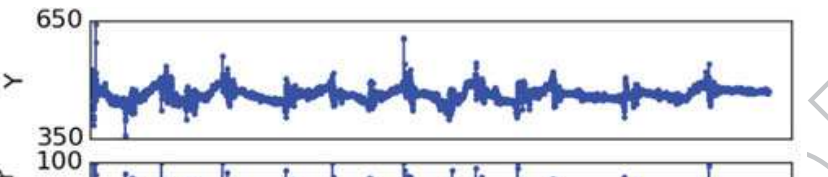

a)

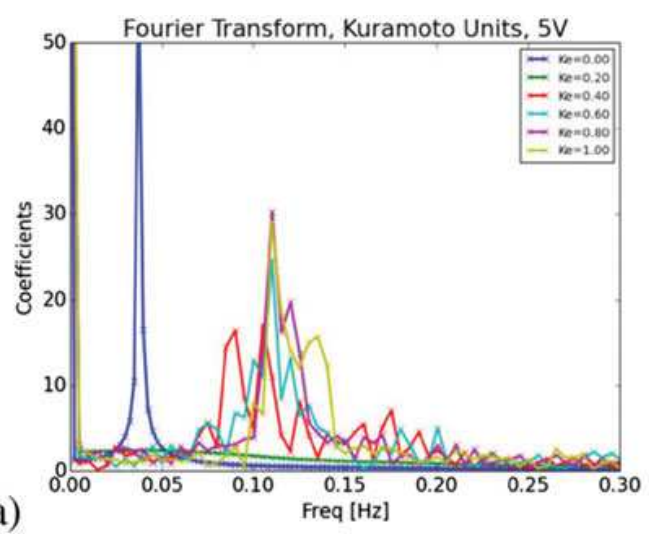

graph in Figure 7 showing the structure $Y$ displacement to the vertical axis, the PD error computed and the motor control on it. It shows that the PD controller rapidly stabilizes its dynamics to the upward posture concerning perturbations, which corresponds more to a reflexive bang-bang controller when the $K_{D}$ coefficient is tuned with a small value relative to $K_{P}$. Although the structure presents a rhythmical pattern, the transitions are abrupt as we do not observe any resonant modes in this configuration (top chart). In this control mode, the motors contribute mostly to shock absorptions, which is less energy-efficient and less compliant. We will study and compare thereinafter other controllers based on oscillators to overcome this problem of smooth control.

\subsection{Kuramoto oscillators control}

Our first experiment with the Kuramoto oscillators consists in studying the interval range of the external coupling parameter KE and see the impact feedback on the internal dynamics of the oscillators when they are coupled to it. We study first the Fourier coefficients of Kuramoto's units when the control is done in a closedloop manner when we make to vary $\mathrm{KE}$ within the interval range $[0 ; 1]$; the motor signal is normalized within the interval range $[0,255]$ and translated into PWM to the motors. We display the result of the Fourier transform in Figure 8(a) and of the dynamics of one motor in Figure $8(\mathrm{~b})$ within the interval range $K E \in[0.15 ; 0.41]$. In our experiments, the internal coupling, $\mathbf{K I}=0$ and the amplitude level of the oscillators, $\mathrm{JE}=1$ are not changed.

When $\mathrm{KE}=0.0$, the oscillators drive the tensegrity structure in a completely open-loop fashion to the

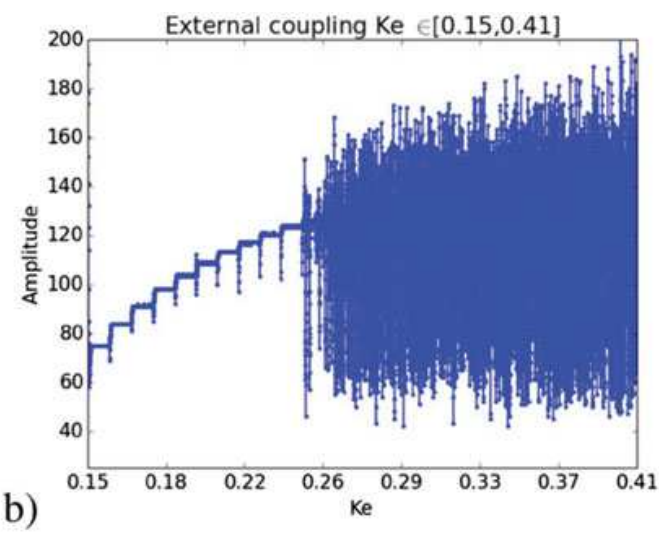

Figure 8. Influence of the external coupling parameter KE on the resonant modes of the tensegrity structure. In (a), we plot the Fourier coefficients with respect to the quantity of feeded back signal injected into the oscillators within the interval range $\mathbf{K E} \in[\mathbf{0 . 0}, \mathbf{1 . 0}]$ from open-loop control to stable closed-loop entrainment. In (b), we plot the details of the dynamics of one oscillator with respect to KE in the interval range $[\mathbf{0 . 1 5}, \mathbf{0 . 4 0}]$ when the system bifurcates from an under-damped state to a rhythmical regime with the resonant frequencies of the tensile structure. 
881

891

a)
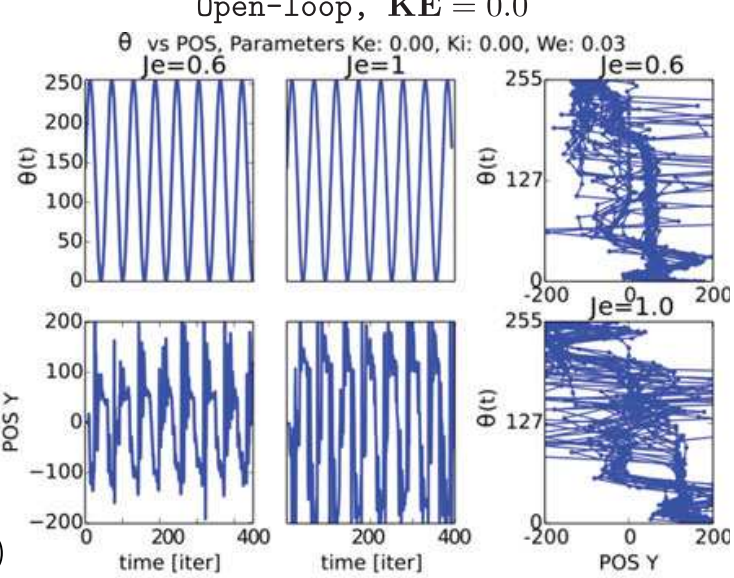

896

901

906

b)
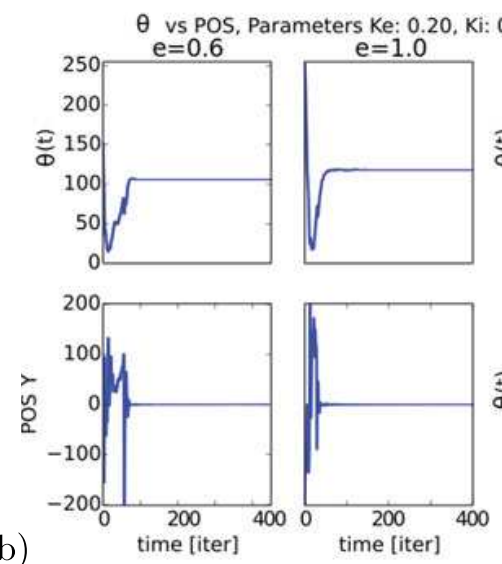

Sync, KE $>0.2$
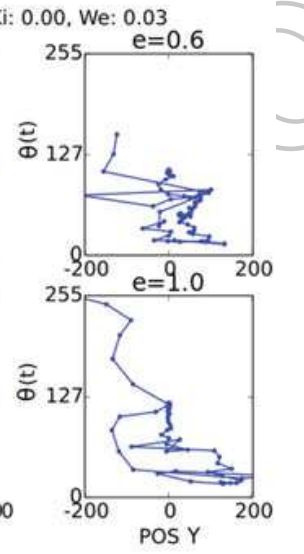

911

916
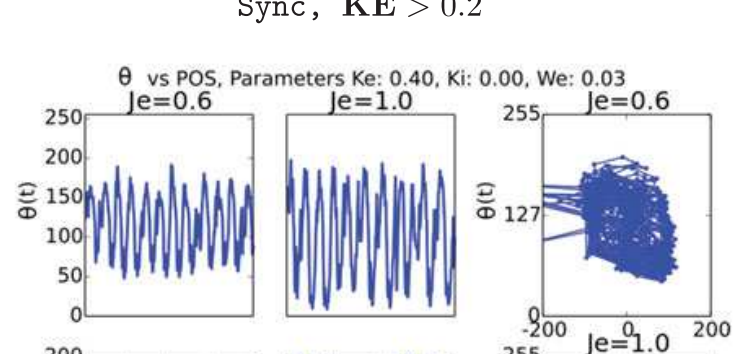

921

c)
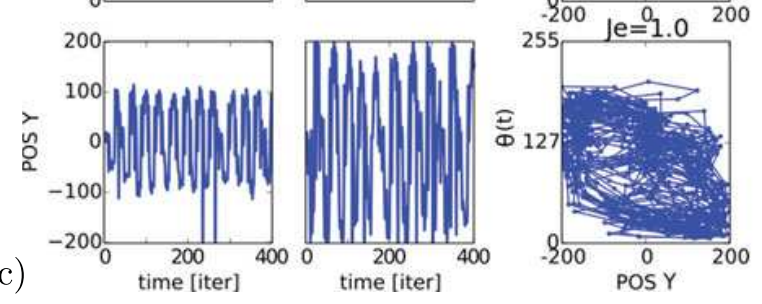

Figure 9. Phase plots for external coupling KE corresponding to three different behavioral patterns and for different amplitudes JE. (a) $\mathbf{K E}=0.0$, the oscillators control in open-loop vertebral column to their intrinsic regime different from the one of the structure. (b) $\mathbf{K E}=0.2$, The oscillators go to a stable point attractor that return back when perturbed. This corresponds to a stable and passive pre-reflexive stage. (c) $\mathbf{K E}>0.2$, the general regime of synchronization is stabilized to generate rhythmical patterns around the center, which vary also depending on the motor force JE. intrinsic frequency $\omega$ of the oscillators. In this stage, the tensegrity structure performs a strong rhythmic motion. At $\mathbf{K E}=0.2$, an interesting behavior occurs in which the oscillators bifurcate to an attractor point, which is an under-damped postural configuration due to the friction of the ball joints. The friction poises the structure to the vertical position. The structure is slowly entrained by a slight feedback control till its immobilization. This posture is in this plot the upward posture with $Y=0$. In this situation, the motors remain in this static posture without any feedback and react to small perturbations: when the structure is slightly pushed, the oscillators act reflexively to return back to the static posture.

Above this value, say for $\mathrm{KE} \geq 0.4$, the oscillators start to be entrained actively by the external dynamics with a signal per noise ratio that depends on KE values. In this stage, the feeded back signal generates stable cyclic motion around the upward position in which, the higher the coupling coefficient, the higher the instabilities of the rhythmical pattern.

To understand in more details what is going on between the two regimes found, we plot the dynamics of the oscillator in the interval range $\mathrm{KE} \in[0.15 ; 0.4]$, see Figure 8(b). In coherence with the results found in Figure 8(a), we observe a point transition only at $\mathrm{KE}=$ 0.25 , which corresponds to a bifurcation diagram of the oscillators dynamics when they start to synchronize to the external dynamics of the body structure and start to mutually influence each other to this particular rhythm. This state corresponds to what is called feedback resonance, a mechanism that drives one system to its resonant frequencies by feedback and for certain coupling strength. This approach is similar to synergistic control $[6,8]$, nonetheless, we characterized this phenomenon in robotics as a way to control postural coordination in different robotic systems [29,37-39].

We analyze in the next paragraph the three cases found depending on the coupling parameters $\{\mathbf{K E}, \mathbf{K I}, \mathbf{J E}\}$.

We display three different phase plots for the three behaviors presented earlier with respect to $\mathrm{KE} \in\{0.0 ; 0.2$; 0.4 , see Figure 9 resp. $(\mathrm{a}-\mathrm{c})$. But to define more precisely the behavior of the system, we add two new conditions to compare with when $\mathbf{J E}=\mathbf{0 . 6}$ and when $\mathbf{J E}=\mathbf{1 . 0}$ that modulate more or less strongly each motor output. The two left columns of top charts indicate the oscillator's phase over time $\vartheta(t)$ given to the motors between $[0 ;+255]$ and the two left column of bottom charts indicate the vertical displacement over time $Y(t)$ or $Y_{\text {err }}$ relative to the vertical axis and normalized between $[-100 ;+100]$, which corresponds to $[-30 ;+30 \mathrm{~cm}]$. The third column in Figure $9(\mathrm{a}-\mathrm{c})$ corresponds to the phase plot of the two variables $Y$ and $\vartheta$, respectively in the 
$x$ and $y$ axis, which describes the temporal dependence of the two variables. These graphs show the plots during 10, s of the internal CPGs dynamics and of the position at the tip of the structure oscillating around the $Y$ axis for $\mathrm{JE} \in[0.6 ; 1.0]$.

In Figure 9(a), the tensegrity structure is totally open-loop driven and forced to follow the oscillators cycle without any feedback. In contrast, the situation in Figure 9(b) corresponds to an under-damped case for $\mathrm{KE}=0.2$ where the oscillators go to a point attractor centered on the neutral position of the structure. Besides, when the coupling term $\mathbf{K E}$ augments above 0.2 as it is the case in Figure 9(c), the Kuramoto oscillators start to be entrained to the phasic regime of the structure. In line with the Fourier analysis in Figure 8, Figure 9 describes 1006 how the coefficient JE affects synchronization and the system behavior as it is for KE. We will present thereinafter in the next section the top-down control done on the global parameters $\{\mathbf{K I}, \mathbf{K E}, \mathbf{J E}\}$ for updating the oscillators to the desired regime.

1011

\subsection{PD control on Kuramoto oscillators}

The two methods presented in the previous Sections 4.3 and 4.4 have different advantages regarding controllability concerning the desired state (resp. the PD controller) and regarding compliance control concerning the desired rhythm (resp. the oscillators). The association of the two controllers type may permit to combine the advantages of the two worlds for generating one controlled entrain-

1021 ment to resonant modes and robust to perturbations. We propose to study this combined controller for the balance control of the tensegrity column in the upward posture by the amplitude modulation of JE on the oscillators based on error feedback $e$. We remind the reader that JE is governed by the equation JE $=K_{P} Y_{\text {err }}+K_{D} \dot{Y}_{\text {err }}$ where $Y_{\text {err }}$ is the horizontal displacement relative to the vertical axis.

We plot in Figure 10 four time series of the structure's stabilization for different pushes of gradual strength with the plot of the $Y$ axis deviation in the top chart, and of the PD error over time in the middle chart and of the motor activity in the bottom chart for two motors in the opposite side. In order to better understand the dynamics, a motion sequence is presented in Figure 11 of a recovery after a stroke and a movie are provided at the address https://sites.google.com/site/embodiedai/currentresearch/tensegrityrobots.

The top-down control strategy used to stabilize the structure is based on the modulation of JE concerning the displacement on the $Y$ axis employed as error feedback. Depending on the perturbation force applied on the structure -, that we characterize in percent from
$5 \%$ to $20 \%$ of relative displacement,- various transitory regimes can be observed till stabilization. This 'resynchronization' process has an impact on the temporal period, the phase and the frequency adaptation.

When a small stroke is applied as in Figure $10(\mathrm{a}, \mathrm{b})$, the PD controller generates small amplitude variations till convergence of the oscillatory regime of the Kuramoto units with the damping of the structure between 10 and $15_{\perp} s$ to its upward posture. The oscillatory regime of the CPGs is similar to an under-damped regime for a spring-mass system with a long lasting transitory regime of a dizain of cycles. For instance, when the perturbation reaches $10 \%$ displacement, the PD controller desynchronize several times and takes several trials to re-stabilize the oscillators and the structure.

In the case of a strong perturbation as it is in Figure 10 (a,d) for $15 \%$ and $20 \%$ perturbations, the PD controller generates at reverse higher amplitude adaptations of the Kuramoto units till absorption of the shock and damping of the structure. The temporal employed is slightly higher in this forced regime with a longer synchronization stage diminution of the oscillators amplitude and phase difference. This behavior of the CPGs is similar to an over-damped regime for a spring-mass system with a faster transitory state of several seconds but after the stabilization of the oscillatory, which takes longer times (around 20 cycles).

In all the situations, the oscillatory system finished with a small vibratory mode around the vertical axis due to the tuning of the PD coefficients, till its return back to a static posture with the release of the motors from cocontraction. Our strategy was efficient in all the studied cases, irrespective of the shock level but with some sensitivity of the PD controller to small perturbations due to its coefficients. These small perturbations made the system to slightly reactivate the oscillations as it is displayed in Figure 10(b) at time $48 \mathrm{~s}$. We can understand from this figure that the external dynamics of the vertebral column excites the oscillators and reactivates their dynamics.

The top-down controller performed on the oscillators permits to modulate more adaptively the strength of the motor synergies relative to the task than the PD controller. To compare the two strategies, we plot the error relative to the upward posture for the PD controller and the PD-controlled oscillators, respectively in Figure 12 $\left(\mathrm{a}_{2} \mathrm{~b}\right)$. The figure shows that the second strategy (PD control + Kuramoto oscillators) exploits more smoothly the motors with less strength applied on them (with a PWM below 150), which is at the price of a larger error variance. This complementary strategy exploits better the body structure and the motor synergies with unrestricted 


$$
\text { perturbation error }<5 \%
$$

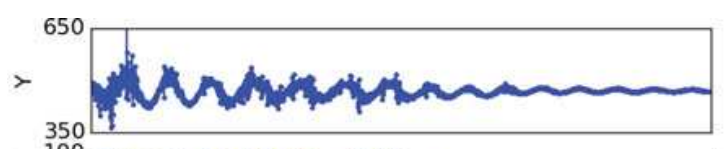

1106

1111

1116

1121$$
\text { a) }
$$
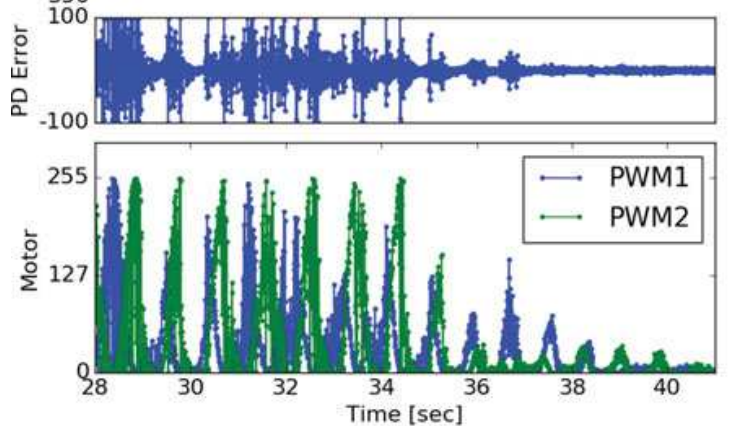

a)

perturbation error $<15 \%$
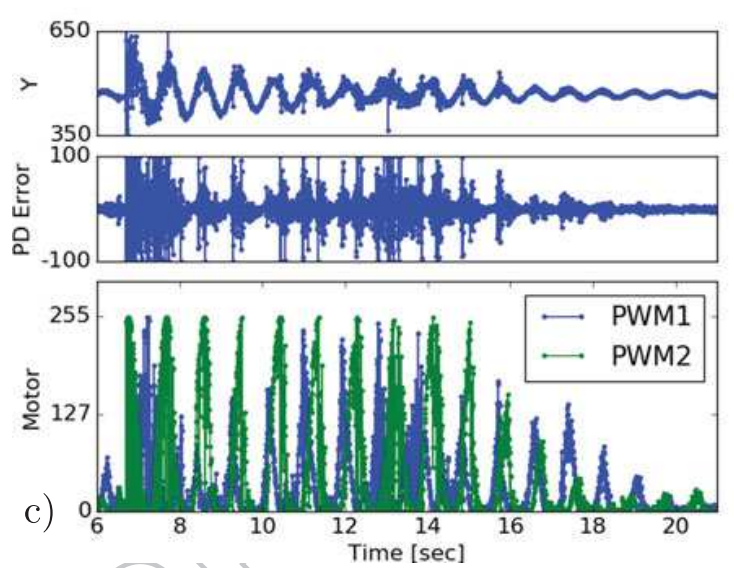

perturbation error $<10 \%$
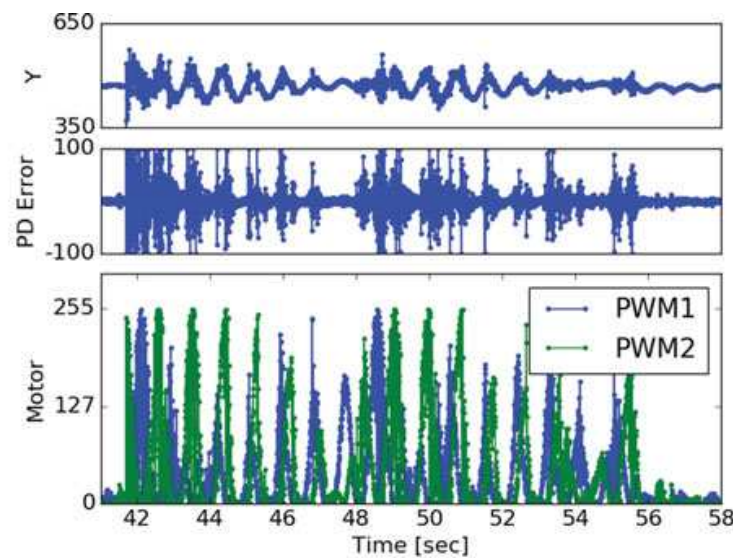

b)

perturbation error $<20 \%$
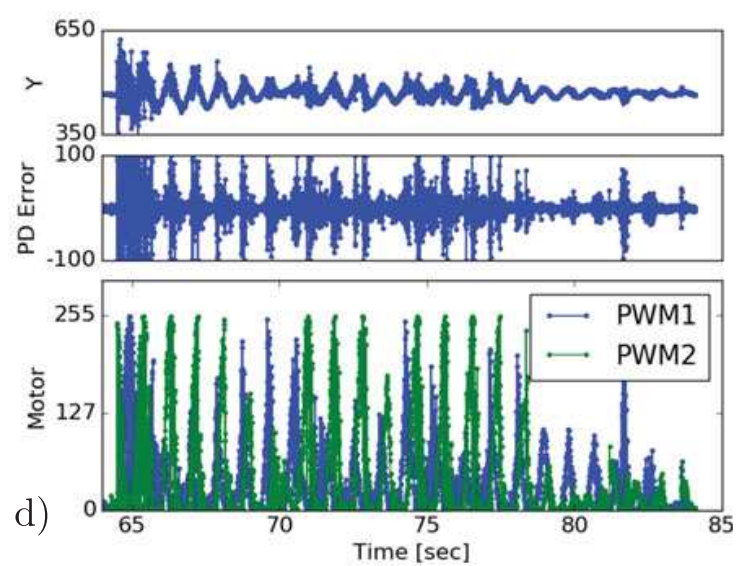

1156

Figure 10. Feedback control of the global parameter JE on the Kuramoto oscillators for various external perturbations. When a strong perturbation is imposed on the structure, an oscillatory regime is established by the top-down controller on the vertebral column to absorb the shock with a high amplitude till their attenuation. This transitory regime depends on the amplitude of the shock and of the velocity of the JE to steer the oscillators and the motors till their release when the column is stable enough to return back and stand at its upward posture. The more JE decays slowly, the longer the transition to a stable regime (under-damped oscillations). The more JE decays fastly, the quicker the transition to a stable regime (over-damped oscillations).

$t=0 s$

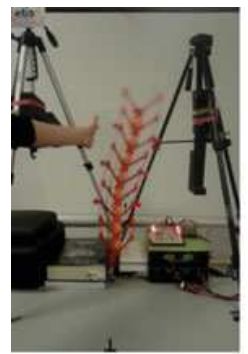

$t=2.4 s$

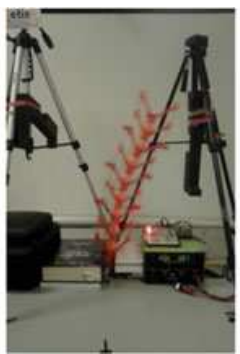

$t=2.8 s$

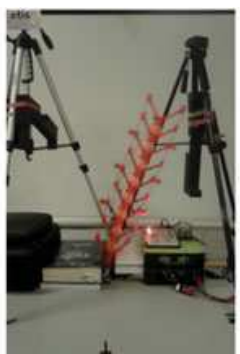

$t=3.2 s$

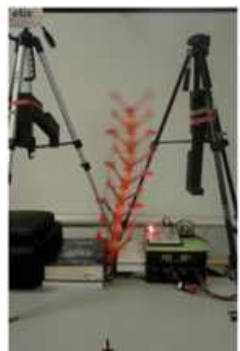

$t=3.6 s$

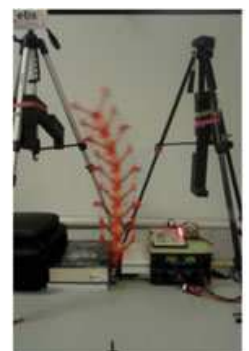

$t=4.4 \mathrm{~s}$

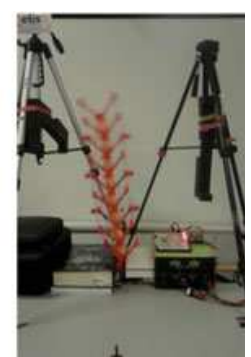

Figure 11. Motion sequence of postural balance after a stroke. A complete movie is provided at the address https://sites.google.com/site/ embodiedai/current-research/tensegrityrobots. 
1221

$$
\text { a) }
$$

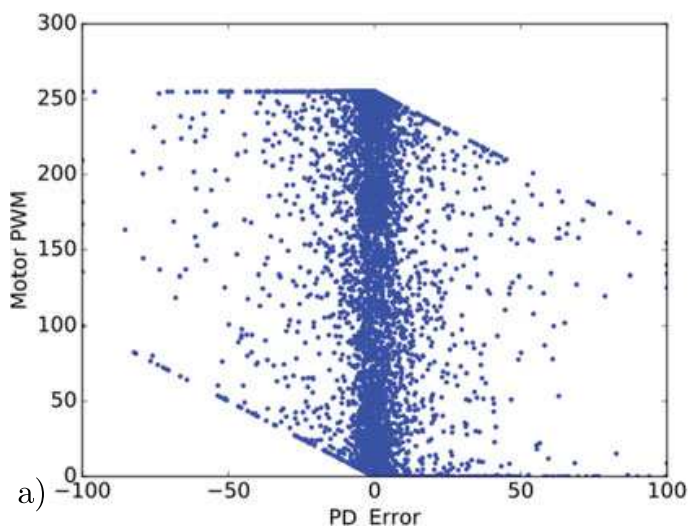

PD Control $\rightarrow$ Kuramoto

1266

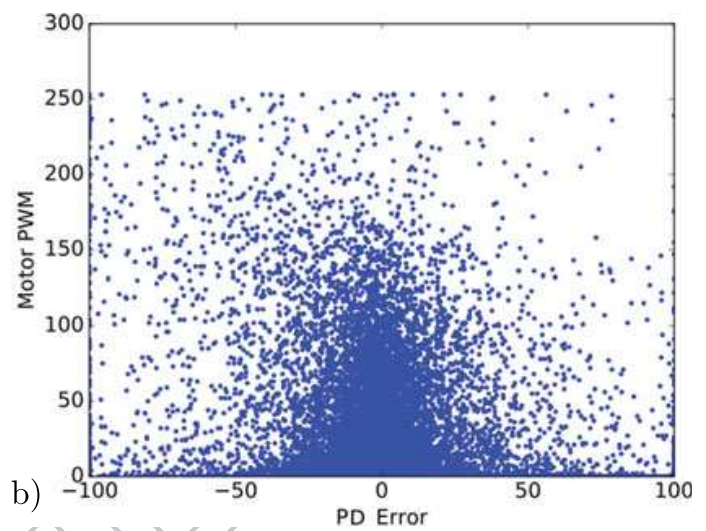

1271

Figure 12. Motor contribution with respect to the control strategy and perturbation. Comparison of motor dynamics between the PD controller in (a) and the PD controller on the Kuramoto oscillators in (b). In (a), the motors are strongly solicited to minimize error drastically from any perturbation. In (b), the motors are less strongly exploited; which has in consequence the loose error. The second strategy permits to exploit better the body structure and its passivity in order to have a more compliant and energy-efficient control on the body structure.

dynamics and loose error, which makes it better suited to absorb any external shocks.

\section{Discussion}

1236

1241

We presented a tensegrity-based model of a vertebral column robot controlled actively with nonlinear oscillators and feedback for rhythmical balance and upward posture. From a control viewpoint, the physics of this complex system requires to adopt a more bio-inspired type of control with loosely and parallely distributed units for adaptation to the body dynamics [28]. In the end, the elastic properties of the tensegrity-based articulated trunk may ease its control [49]; i.e. its morphological control [50-52]. The CPGs in the spinal cord are local neural units that can generate a rhythmical pattern even without any feedback. Their activity, however, is always under the local feedback control of muscle spindle signals and the global feedback control of neuromodulators at the spinal circuits level or at the higher level. These feedback loops make these units embodied in the physics of the structure and contingent to a global coordination at the task level.

We use Kuramoto oscillators to entrain the vertebral column to its own rhythms and to synchronize them dynamically to the structure's resonant frequencies thanks to the IMU unit. This phenomenon is known as feedback resonance $[29,37,53,54]$ and is used as a strategy to control the vertebral column to its resonant frequencies for shock absorption and postural balance. The tensile robot presents interesting damping properties that makes it easy to stabilize at the upward configuration, either passively or actively $[55,56]$. This is in line with observations found on biological systems and humans on the importance of an actuated head and flexible trunk to body balance $[18,31,45]$ and passive walkers $[49,57]$. We extended then the oscillator network to adaptive control with the use of feedback error for dynamical synchronization of the structure to the upward posture. This model is in line with other biological models of neurons for robotics that includes reciprocal, inhibitory and oscillatory modes $[39,40,58,59]$. However, our neural controller is combining PD control+oscillators in order to produce controlled rhythmical patterns, which is original and appears not used by other teams. Our feedbackdriven oscillatory network controls global parameters for the dynamical entrainment of the resonant frequencies so that it can serve for discrete phase resetting [47] or modulated attenuation/amplification; this approach is in line with the proposal by Bizzi of motor synergies [34].

Our model presents also similar properties with $[8,18]$ and provides realistic predictions of postural sway movements during erected head tracking. In our system, bifurcation and nonlinearities emerge from online optimization due to the combination of phase synchronized oscillators and PD control, which is difficult to achieve with offline models. Adaptation and recovery are possible only when synchronization is done around the frequencies of the body dynamics and found during the test phase. Further versions may examine the online discovery and learning of the structural parameters of the system and of the task, such as $\omega$ and the coupling parameter $\{\mathrm{KE}, \mathrm{KI}, \mathrm{JE}\}$ and $Y_{\text {err }}$, by a top-down neural network or by a pseudo-inverse matrix that store and anticipate these information. 
We show that tensile structures present interesting properties for the design of soft and bio-inspired robots with the use of replicative elements to insure a redundancy of the global behavior at the macroscale level and flexibility with the many degrees of freedom of each element. These structures are lightweights and pre-tensed, which make them physically robust to shocks even passively as they distribute their tension on all their elements. Furthermore, their stiffness can be linearly controlled with the co-contraction of the motors to switch from a flexible behavior to a rigid one. The dimensionality of the system makes it a complex system and the way to control it requires to exploit its passive dynamics and to entrain the controllers to its resonant frequencies for upward balance or rhythmical motion.

Starting from this tensegrity-based vertebral column robot, it is natural then to think to design other body parts based on tensegrity $[13,60]$. We will attempt to go further in that direction with a more complex body and behaviors such as postural coordination and walking

1341 [38]. At now, we have employed only one IMU unit in the top vertebral column to allow head-centered coordinated movements [18] as it is known that the vestibular system, perceiving rotational velocities and linear accelerations, uses this information to generate a unified inertial reference frame, centered in the head that allows whole-body coordinated movements and head-oriented locomotion $[61,62]$. In future works, we will employ more IMU units for each segment to have proprioception at the body level.

Finally, as an educational tool, the scalability of tensegrity structures fulfills the requirements for testing ideas with low-cost and multi-disciplinary platforms at the community level for exploration and experimentation in robotics for Research, as well as in Art and Education. For instance, tensile structures can be $3 \mathrm{D}$ printed and can be highly replicable, which can be interesting for the DoIt-Yourself community. In the complement of this paper, we provide a website of the project with links for downloading freely the tensegrity modules for building it and to inspire and iterate on the project to one's own. Their 1361 overall robustness and lightweight can be put forward in comparison to most robots, which are still fragile and expensive products to design and repair. The ecology of the body morphology changes also the way control in force and precision is done and requires to understand biological control and to rethink it for robots.

\section{Acknowledgments}

\section{Disclosure statement}

No potential conflict of interest was reported by the authors.

Q5

\section{Funding}

This research project was partially funded by chaire d'Excellence

CNRS-UCP, the project Labex MME-DII (ANR11-LBX-0023- Q6. 01) and EQUIPEX-ROBOTEX (CNRS).

\section{References}

[1] Pfeifer R, Lungarella M, Iida M. Self-organization, embodiment, and biologically inspired robotics. Science. 2007;318:1088-1093.

[2] McEvoy M, Correll N. Materials that couple sensing, actuation, computation, and communication. Science. 2007;318:1088-1093.

[3] Bernstein N. The coordination and regulation of movements. Oxford: Pergamon Press; 1967.

[4] Todorov E. Optimality principles in sensorimotor control. Nat Neurosci. 2004;7(9):907-915.

[5] Bizzi E, Giszter S, Loeb E, et al. Modular organization of motor behavior in the frog's spinal cord. Trends Neurosci. 1995;18:442-445.

[6] Kelso JS. Dynamic patterns: The self-organization of brain and behavior. Cambridge, MA: MIT Press; 1995.

[7] Kelso JS, Haken H. Synergetics of brain and behavior. In What is Life? The Next Fifty Years. 1995; 137-160.

[8] Bardy B, Oullier O, Bootsma R, et al. The dynamics of human postural transitions. J Exper Psychol Hum Percept Perform. 2002;28:499-514.

[9] Ly O, Lapeyre M, Oudeyer P. Bio-inspired vertebral column, compliance and semi-passive dynamics in a lightweight robot. International Conference on Robots and Systems, San Francisco. 2011. p.1-8.

[10] Nakajima K, Hauser H, Kang R, et al. A soft body as a reservoir: case studies in a dynamic model of octopus-inspired soft robotic arm. Front Comput Neurosci. 2013;7(91).

[11] Zhao Q, Sumioka H, Nakajima K, et al. Spine as an engine: effect of spine morphology on spine-driven quadruped locomotion. Adv Robot. 2014;28(6):367-378.

[12] Wei X, Wang C, Long Y, et al. The effect of spine on the bounding dynamic performance of legged system. Adv Robot. 2015;29(15):973-987.

[13] Flemons T. The bones of tensegrity. http://wwwintension designscom/bones_of_tensegrity. 2012

[14] Bizzi FEClarac. Motor systems. Curr Opin Neurobiol. 1999;9(6):659-662.

[15] Marder E, Calabrese R. Principles of rhythmic motor pattern production. Physiol Rev. 1996;76:687-717.

[16] Calabrese R. Oscillation in motor pattern-generating networks. Curr Opin Neurobiol. 1995;5:816-823.

[17] Bardy B, Marin L, Stoffregen T, et al. Postural coordination modes considered as emergent phenomena. Exp Psychol Hum Percept Perform. 1999;25:1284-1301.

[18] Bonnet V, Ramdani S, Fraisse P, et al. A structurally optimal control model for predicting and analyzing human postural coordination. J Biomech. 2011;44: 2123-2128.

.


1431 [19] Taga G. A model of the neuro-musculo-skeletal system for human locomotion. i. emergence of basic gait. Biol Cybern. 1995;73(2):97-111.

[20] Der R, Hesse F, Martius G. Learning to feel the physics of a body. Proceedings of the International Conference on Computational Intelligence for Modelling, Control and Automation (CIMCA 06); Washington, DC: IEEE Computer Society. 2005. p. 252-257.

Q9.

[21] Fuller RB. Synergetics: Explorations in the geometry of thinking. Scribner. 1975

[22] Snelson K. Continuous tension, discontinuous compression structures. United States patent 3169611. 1965

1441 [23] Paul C, Valero-Cuevas F, Lipson H. Design and control of tensegrity robots for locomotion. IEEE Trans Robot. 2006;22(5):878-980.

[24] Bliss T, Werly J, Iwasaki T, et al. Experimental validation of robust resonance entrainment for cpg-controlled tensegrity structures. IEEE Trans Control Syst Technol. 2008;21:666-678.

1446

[25] Tietz B, Carnahan R, Bachmann R, et al. Tetraspine: Robust terrain handling on a tensegrity robot using central pattern generators. IEEE/ASME International Conference on Advanced Intelligent Mechatronics (AIM); ${ }_{451}$ Q10 2013. p. 261-267.

[26] Hauser H, Ijspeert A, Fchslin R, et al. Towards a theoretical foundation for morphological computation with compliant bodies. Biol Cybern, 2011;105(xx):355-370.

[27] Hauser H, Ijspeert A, Fchslin R, et al. The role of feedback in morphological computation with compliant bodies. Biol Cybern. 2012;106(10):595-613.

1456 [28] Caluwaerts K, Despraz J, Sabelhaus A, et al. Design and control of compliant tensegrity robots through simulation and hardware validation. J R Soc Interface. 2014;11:20140520.

[29] Pitti A, Lungarella M, Kuniyoshi Y. Quantification of emergent behaviors induced by feedback resonance of chaos. Recent Adv Artif Life: Adv Nat Comput. 2005;3(15):199-213.

[30] Ingber D, Wang N, Stamenovi D. Tensegrity, cellular biophysics, and the mechanics of living systems. Rep Prog Phys. 2014;046603(77):48-57.

1466 [31] Turvey M, Fonseca S. The medium of haptic perception: a tensegrity hypothesis. J Mot Behav. 2014;46(3):143-187.

[32] Levin S. The tensegrity-truss as a model for spine mechanics: biotensegrity. J Mech Med Biol. 2002;2: 375-388.

[33] Strogatz S. Sync: the emerging science of spontaneous

1471 [34] Bizzi E, Cheung V, d’Avella A, et al. Combining modules for movement. Brain Res Rev. 2008;57(1):125-133.

[35] Ting L. Dimensional reduction in sensorimotor systems: a framework for understanding muscle coordination of posture. Prog Brain Res. 2007;165:299-321.

1476

[36] Allen J, Ting L. Why is neuromechanical modeling of balance and locomotion so hard? Chapter 7. J Mot Behav. 2016;7:197-223.

[37] Pitti A, Lungarella M, Kuniyoshi Y. Exploration of natural dynamics through resonance and chaos. Proceedings of the 9th Conference on Intelligent Autonomous Systems;

\section{Q11 2006. p. 558-565}

[38] Pitti A, Lungarella R, Kuniyoshi Y. Generating spatiotemporal joint torque patterns from dynamical synchronization of distributed pattern generators. Front Neuro-Robotics. 2009;3(2). 10.3389/neuro.12.002.2009.

[39] Pitti A, Niiyama R, Kuniyoshi Y. Creating and modulating rhythms by controlling the physics of the body. Auton Robots. 2010;23(8):317-329.

[40] Melnyk A, Henaff P. Bio-inspired plastic controller for a robot arm to shake hand with human. Proceedings of IEEE International Conference on Electronics and Nanotechnology, Kiev, Ukraine; 2016. p. 163-168

[41] Ijspeert A, Nakanishi J, Schaal S. Learning attractor landscapes for learning motor primitives. Cambridge, MA: MIT Press; 2003.

[42] Ijspeert A. Central pattern generators for locomotion control in animals and robots: a review. Neural Netw. 2008;21(4):642-653.

[43] Frumar A, Zhou Y, Xie Y, et al. Tensegrity structures with 3d compressed components: development, assembly and design. J Int Assoc for Shell and Spatial Struct. 2009;161(2):99-110.

[44] Tinkercad. https://tinkercad.com/things/6ibmnx721ak. 2016.

[45] Bardy B, Oullier O, Lagarde J, et al. On perturbation and pattern co-existence in postural coordination dynamics. J Mot Behav. 2007;39:326-334.

[46] Geyer H, Herr H. A muscle-reflex model that encodes principles of legged mechanics produces human walking dynamics and muscle activities. IEEE Trans Neural Systems Rehabilitation Eng.2010.

[47] Nakanishi J, Morimoto J, Endo G, et al. An empirical exploration of phase resetting for robust biped locomotion with dynamical movement primitives. International Conference on Intelligent Robots and Systems; 2004. p. 919-924.

[48] Hoinville T, Tapia Siles C, Henaff P. Flexible and multistable pattern generation by evolving constrained plastic neurocontrollers. Adapt Behav. 2011;19(3):187-207.

[49] Alexander M. Walking made simple. Science. 2005;308 (5718):58-59.

[50] Pfeifer R, Bongard J. How the body shapes the way we think, a new view of intelligence. Bradford Books; 1999.

[51] Pfeifer R, Gomez G. Morphological computation connecting brain, body, and environment - creating brain-like intelligence: from basic principles to complex intelligent systems. Lecture Notes in Artificial Intelligence, Creating Brain-Like Intelligence, Vol. 5436; Berlin: Springer; 2009. p.66-83.

[52] Pfeifer R, Pitti A. La révolution de l'intelligence du corps (French). Manuella Editions; 2012.

[53] Fradkov A. Exploring nonlinearity by feedback. Phys D: Nonlinear Phenom. 1999;128(2):159-168. Available from: http://www.sciencedirect.com/science/article/pii/ S0167278998003224.

[54] Fradkov AL. Investigation of physical systems by means of feedback. Autom Remote Control. 1999;60(3).

[55] Iida F, Pfeifer R. Self-stabilization and behavioral diversity of embodied adaptive locomotion. Embodied artificial intelligence. In Iida et al., Editors, Lecture Notes in Computer Science/Artificial Intelligence; Vol. 3139; 2004. Berlin: Springer: Berlin.p. 119-128

[56] Ikemoto S, Nishigori Y, Hosoda K. Advantages of flexible musculoskeletal robot structure in sensory acquisition. Artif Life Robot. 2012;17(1):63-69. 
1541 [57] Laumond JP, Benallegue M, Carpentier J, et al. The YoyoMan. International Symposium on Robotics Research (ISRR); Sep.; Sestri Levante, Italy; 2015.

[58] Amrollah E, Henaff P. On the role of sensory feedbacks in Rowat-Selverston cpg to improve robot legged locomo-

Q18 tion. Front Neurorobot. 2010;4.
[59] Nassour J, Hénaff P, Benouezdou F, et al. Multi-layered multi-pattern CPG for adaptive locomotion of humanoid robots. Biol Cybern. 2014;108(3):291-303. Available from: http://view.ncbi.nlm.nih.gov/pubmed/24570353.
[60] Fujita K, Yonekura S, Nishikawa S, et al. Environmental and structural effects on physical reservoir computing with tensegrity. Proceedings of the 5th IIAE International Conference on Intelligent Systems and Image Processing; 2017. p. 1-8.

[61] Berthoz A. The brain's sense of movement. Harvard University Press; 2000.

[62] Falotico E, Cauli N, Kryczka P, et al. Head stabilization based on a feedback error learning in a humanoid robot. Auton Robots. 2016;1-17.

Q21 